\title{
Author Correction: Regional ambient temperature is associated with human personality
}

Wenqi Wei, Jackson G. Lu, Adam D. Galinsky, Han Wu, Samuel D. Gosling, Peter J. Rentfrow, Wenjie Yuan, Qi Zhang, Yongyu Guo, Ming Zhang, Wenjing Gui, Xiao-Yi Guo, Jeff Potter, Jian Wang, Bingtan Li, Xiaojie Li, Yang-Mei Han, Meizhen Lv, Xiang-Qing Guo, Yera Choe, Weipeng Lin, Kun Yu, Qiyu Bai, Zhe Shang, Ying Han and Lei Wang*

Correction to: Nature Human Behaviour https://doi.org/10.1038/s41562-017-0240-0 (2017); published online 27 November 2017.

In the Supplementary Information file originally published for this Letter, in three places 'conscientiousness' was mistakenly written 'contentiousness', and on page 11, 'second' should have read 'secondary', and 'third' should have read 'tertiary'. These errors have been corrected.

Published online: 1 December 2017

https://doi.org/10.1038/s41562-017-0275-2 